\title{
QUALIDADE DE VIVA DE IDOSOS ATENDIDOS EM CENTRO DE LAZER: ESTUDO TRANSVERSAL
}

Rafael André da Silva ${ }^{1}$ Sabrina da Silva Brasil ${ }^{1}$ Andryelli Aires de Moraes ${ }^{1}$ Nébia Maria Almeida de Figueiredo ${ }^{1}$ Aline Coutinho Sento Sé ${ }^{1}$ Alexandre Sousa da Silva Teresa Tonini ${ }^{1}$ Wiliam César Alves Machado ${ }^{1}$

\author{
https://orcid.org/0000-0001-7183-8415 \\ https://orcid.org/0000-0002-6820-3141 \\ https://orcid.org/0000-0003-0447-1160 \\ http://orcid.org/0000-0003-0880-687X \\ https://orcid.org/0000-0001-9301-0379 \\ https://orcid.org/0000-0002-5573-4111 \\ https://orcid.org/0000-0002-5253-2485 \\ https://orcid.org/0000-0002-2880-0144
}

Objetivo: Analisar a qualidade de vida de idosos que participam de atividades inclusivas em um centro de lazer na Cidade do Rio de Janeiro. Metodologia: estudo transversal, realizado no período de setembro de 2014 a julho de 2015 , com 69 idosos cadastrados em Centro de Lazer da Cidade do Rio de Janeiro, Brasil. Utilizou-se para coleta de dados os instrumentos WHOQOLBREF, WHOQOL-OLD e questionário sócio demográfico. Resultados: No instrumento WHOOOL-BREF verificou-se que o domínio com a maior pontuação média foi o domínio Psicológico, já no WHOOOL-OLD foi à faceta Funcionamento do Sensório. Constatou-se que as variáveis religião e uso de medicamentos exerceram influência de forma significativa sobre a qualidade de vida dos indivíduos estudados. Conclusão: Este estudo fornece subsídios para aprimorar a atuação dos profissionais de saúde interessados em executar práticas proativas de promoção de qualidade de vida e saúde dos idosos.

Descritores: Qualidade de vida; Assistência a Idosos; Atividades de Lazer; Centros de Convivência e Lazer.

\section{QUALITY OF LIVE ELDERLY IN ASSISTED LEISURE CENTER: CROSS-SECTIONAL STUDY}

Objective: To analyze the quality of life of elderly people who participate in inclusive activities in a leisure center in the city of Rio de Janeiro. Methodology: cross-sectional study, conducted from September 2014 to July 2015, with 69 elderly registered at the Leisure Center of the City of Rio de Janeiro, Brazil. For data collection, the instruments WHOQOL-BREF, WHOQOL-OLD and socio-demographic questionnaire were used. Results: In the WHOOOL-BREF instrument it was found that the domain with the highest average score was the Psychological domain, while in the WHOOOL-OLD it was the Sensory Functioning facet. It was found that the variables religion and drug use had a significant influence on the quality of life of the individuals studied. Conclusion: This study provides support to improve the performance of health professionals interested in performing proactive practices to promote quality of life and health of the elderly.

Descriptors: Quality od Life: Old Age Assistance; Leisure Activities; Centers of Connivance and Leisure.

\section{CALIDAD DE VIDA DE ANCIANOS ATENDIDOS EN CENTRO DE OCIO: ESTUDIO TRANSVERSAL}

Objetivo: analizar la calidad de vida de las personas mayores que participan en actividades inclusivas en un centro de ocio en la ciudad de Río de Janeiro. Metodología: estudio transversal, realizado entre septiembre de 2014 y julio de 2015 , con 69 personas mayores registradas en el Centro de Ocio de la ciudad de Río de Janeiro, Brasil. Para la recolección de datos, se utilizaron los instrumentos WHOQOL-BREF, WHOQOL-OLD y el cuestionario sociodemográfico. Resultados: en el instrumento WHOQOL-BREF se descubrió que el dominio con el puntaje promedio más alto era el dominio psicológico, mientras que en el WHOOOL-OLD era la faceta del funcionamiento sensorial. Se encontró que las variables religión y uso de drogas tuvieron una influencia significativa en la calidad de vida de los individuos estudiados. Conclusión: Este estudio brinda apoyo para mejorar el desempeño de los profesionales de la salud interesados en realizar prácticas proactivas para promover la calidad de vida y la salud de los ancianos.

Descriptores: Calidad de Vida; Assistencia a los Ancianos; Actividades Recreativas; Centros de Ocio y Convivencia. 


\section{INTRODUÇÃO}

Em 2012, um estudo do IBGE ${ }^{(1)}$ revelou elevação no índice de envelhecimento da população: de $31,7 \%$, no ano de 2001 , para 51,8\%, no ano de 2011. Este aumento da longevidade requer planejamento no âmbito das políticas públicas para atender às demandas por serviços e atendimentos das necessidades de saúde e inclusão social dos idosos no Brasil.

O processo de envelhecimento populacional(2) está diretamente ligado ao aumento gradual da expectativa de vida. Entretanto, a saúde e a qualidade de vida dos idosos, mais que em outros grupos etários, sofrem influência de fatores físicos, psicológicos, sociais e culturais.

Contudo, o aumento na expectativa e qualidade de vida (QV) dos idosos, não estariam associadas exclusivamente à evolução tecnológica e das ciências de saúde, mas, sobretudo, na participação ativa no cuidado da própria saúde, tais como estimulados em grupos de convivência de idosos, que não se limita apenas às atividades físicas e de lazer ${ }^{(3)}$.

A criação de ambientes propícios ao desenvolvimento de atividades na modalidade centros de convivência e novas formas para envelhecer é uma realidade relativamente nova no contexto brasileiro. Esse movimento começou no início das décadas de 1960 e 1970, quando alguns teóricos e profissionais comprometidos com o processo de envelhecimento começaram a se propor a possibilidade de implementar programas de caráter sociocultural e lúdico para a população idosa ${ }^{(4)}$.

No final dos anos 90, a Organização Mundial de Saúde - $(\mathrm{OMS})^{(5)}$ substituiu a expressão envelhecimento saudável por envelhecimento ativo, definindo esse conceito como a otimização das oportunidades de saúde, participação e segurança com o objetivo de melhorar a QV na medida em que as pessoas ficam mais velhas.

Sabe-se que o envelhecimento é um fenômeno extremamente complexo ${ }^{(2.3)}$, causado por diversos fatores que produzem tendências e consequências das mais diversas. Faz mister destacar que a promoção do envelhecimento ativo envolve a conquista de elementos positivos para a OV, permitindo que os indivíduos percebam o seu potencial para o bem-estar físico, social e mental ao longo do curso da vida e que participem da sociedade de acordo com suas necessidades, desejos e capacidades ${ }^{(6)}$

Existem diversas mudanças que ocorrem de forma natural nesse processo de envelhecimento e que interferem de forma significativa na capacidade funcional dos indivíduos, tais como, modificações na adiposidade corporal, na força muscular, na capacidade aeróbica, na flexibilidade, entre outras ${ }^{(7)}$. Mudanças que devem ser monitoradas e postergados seus efeitos comprometedores da OV dos idosos que participam de atividades nos centros de lazer(8).
Há entendimento de que a qualidade de vida é um construto definido de vários modos, pois aspectos culturais, éticos, religiosos e pessoais influenciam a forma como ela é percebida e suas consequências. Apesar das diferentes definições para o termo, existe concordância entre grande parte dos autores de que, para avaliar OV, é necessária a utilização de abordagem multidimensional(7-9).

É importante ressaltar a complexidade de se avaliar a OV dos idosos, pois a mesma depende de diversos determinantes ou indicadores de bem-estar na velhice ${ }^{(2)}$, tais como: Longevidade, saúde biológica, saúde mental, satisfação, controle cognitivo, competência social, produtividade, atividade, eficácia cognitiva, status social, renda, continuidade de papéis familiares, ocupacionais e de relações informais com amigos. No caso deste estudo, esses parâmetros serão coletados por meio dos instrumentos Whoqol-Bref e Whoquol-Old.

A participação em atividades grupais minimiza o sentimento de solidão dos idosos, mesmo que muitos deles vivam acompanhados de familiares. Nesse ponto, faz mister ressaltar que a idade é um fator determinante para o declínio cognitivo(10). Em compensação, a atividade física regular contribui para melhora da qualidade e expectativa de vida do idoso, devendo ser estimulada ao longo da vida e desenvolvida em programas e ações públicas voltadas para a população idosa(11), a exemplo do instituído no cenário deste estudo.

Isto posto, considerando o aumento mundial da longevidade e a necessidade de que seja garantido envelhecimento saudável da população, o presente estudo tem como objetivo analisar a qualidade de vida de idosos que participam de atividades físicas, recreativas, de educação em saúde e intelectuais em centro de lazer.

\section{METODOLOGIA}

\section{Tipo de estudo}

Trata-se de um estudo transversal, descritivo, com abordagem quantitativa, realizado com idosos que participam efetivamente de atividades inclusivas, oferecidas em programa público focado na promoção do bem-estar, qualidade de vida e envelhecimento saudável de idosos que residem no Município do Rio de Janeiro.

\section{Cenário do estudo}

A pesquisa foi realizada na Universidade Aberta da Terceira Idade-UnATI/UERJ que iniciou suas atividades no ano de 1993 como um núcleo da Sub-reitoria de assuntos comunitários da UERJ, tendo programações voltadas para o público com idade mínima de 60 anos, de forma totalmente gratuita, visando melhorar a qualidade de vida dessa parcela da população. 


\section{Caracterização da amostra}

A amostra foi obtida por conveniência e contou com 69 idosos que realizam atividades no programa. A amostragem por conveniência foi utilizada devido ao fato de se tratar da aplicação de instrumentos extensos para um grupo de idoso.

As caracteristicas da amostra foram: 50 participantes do sexo feminino $(72,46 \%)$ e 19 do sexo masculino $(27,54 \%)$; 21 participantes na faixa etária entre 60 e 69 anos (30\%); 31 entre 70 e 79 anos (45\%) e 17 participantes com 80 ou mais anos de idade (25\%). Quanto ao estado conjugal, 23 participantes declararam-se casados (34\%), já entre os 46 (66\%) não casados, 24 são viúvos, 11 solteiros, 8 divorciados e 2 não informaram nenhuma das opções anteriores.

Com relação a escolaridade, 17 possuem ensino inferior ou equivalente ao fundamental completo (25\%), 25 participantes com ensino médio completo (36\%) e 27 com ensino superior completo (39\%). Todos os participantes declararam ter uma religião, sendo 42 católicos (62,69\%), 15 espíritas (22,39\%), 5 evangélicos $(7,46 \%)$ e 5 outras denominações religiosas $(7,46 \%)$.

Quanto a presença de doenças crônicas não transmissiveis 42 (61\%) declararam possuir, enquanto 27 negam, entretanto 55 informam fazer uso regular de medicamentos (79\%).

$\mathrm{Na}$ coleta dos dados foram utilizados os instrumentos WHOOOL-BREF e WHOOOL-OLD, que são instrumentos já validados e amplamente utilizados quando se objetiva pesquisar sobre a qualidade de vida, contudo o segundo instrumento aplica-se especificamente a idosos, além destes, foi utilizado um questionário estruturado com questões relacionadas aos aspectos demográficos e condições de saúde.

\section{Critérios de inclusão e exclusão}

Foram incluidos no estudo, os idosos com idade igual ou superior a 60 anos de idade, de ambos os sexos, que obrigatoriamente sejam usuários efetivos do Centro de Lazer selecionado.

Foram considerados usuários efetivos do centro de lazer todos aqueles individuos que estão devidamente matriculados na UnATI/UERJ, independente do tempo de participação nas atividades.

Foram excluídos do estudo os idosos que estavam frequentando as atividades do Centro de Lazer pela primeira vez, além daqueles que se recusarem a assinar o termo de consentimento livre e esclarecido e os que possuíam alguma condição que os impedisse de responder as questões, como por exemplo déficit cognitivo que inviabilizava o entendimento adequado das questões dos instrumentos. Essa incapacidade era aferida por relato verbal do acompanhante.

\section{Procedimentos de coleta dos dados}

A coleta de dados foi realizada durante o 2 o semestre do ano de 2014 e 1 - semestre do ano de 2015. Amesmafoirealizada em ambiente reservado, que permitia o preenchimento dos formulários com a devida atenção e privacidade.

Iniciou-se a coleta de dados no dia 10/09/2014. As atividades na UnATI/UERJ ocorrem de segunda à sexta das 8 horas às 17 horas, sendo a coleta de dados realizada em dias e horários alternados. Na primeira semana ocorreu às segundas, quartas e sextas de 8 às 13 horas, na semana seguinte às terça e quinta de 13 às 17, na terceira semana às segundas, quartas e sextas de 13 às 17, na quarta semana terças e quintas de 8 às 13 horas, e assim sucessivamente na tentativa de abranger pessoas de todos os horários e atividades. No dia 06/07/2015 a coleta de dados foi finalizada.

Organização e análise dos dados

Os dados coletados pelos três instrumentos (WHOOOLBREF, WHOQOL-OLD e questionário sociodemográfico) foram organizados em planilha eletrônica e posteriormente analisados no programa computacional $R$ na versão 3.2.3.

Após análise exploratória, foram realizados testes de Kruskal-Wallis para comprovar a existência de diferenças estatisticamente significativas. Optou-se por este teste pois nem todas as facetas ou domínios apresentaram distribuição Gaussiana e este fato foi comprovado pelo teste de normalidade de Shapiro-Wilk. Em todas as análises adotou-se nivel de significância de 5\%.

\section{Procedimentos éticos}

O estudo foi desenvolvido consoante com as disposições da Resolução 466/2012 do CNS, e Resolução № 510, de 7 de abril de 2016, que dispõe sobre as normas aplicáveis a pesquisas em Ciências Humanas e Sociais cujos procedimentos metodológicos envolvam a utilização de dados diretamente obtidos com os participantes ou de informações identificáveis ou que possam acarretar riscos maiores do que os existentes na vida cotidiana. Aprovado pelo Comitê de Ética em Pesquisa da Universidade Federal do Estado do Rio de Janeiro (UNIRIO), aprovado pelo parecer no 724.631 , de 21 de julho de 2014, CAAE 33585214.6.0000.5285.

Foi trabalhado, junto com os participantes, o Termo de Consentimento Livre e Esclarecido (TCLE) e, após a sua assinatura e entrega de uma das vias para o participante, foram realizadas as entrevistas. No decorrer da pesquisa, todas as medidas em relação ao sigilo e confidencialidade dos participantes, assim como sua autonomia, foram asseguradas, conforme os princípios éticos das Resoluções já citadas. 


\section{RESULTADOS}

Em complemento aos dados de caracterização da amostra, é importante destacar outros aspectos relevantes para a análise da qualidade de vida dos participantes deste estudo. Quanto à situação profissional, 96\% estão fora do mercado de trabalho e declararam-se aposentados e/ou pensionistas e 2 estão empregados. Com relação a faixa de renda própria 20 recebem de 1 a 3 salários mínimos (29\%), 14 recebem 4 ou mais (20\%) e 35 recusaram-se a responder.

No que concernente ao tempo de participação nas atividades no grupo do centro de lazer, 9 informaram que participam a menos de 1 ano (13\%), 36 afirmam ter tempo de participação no grupo entre 1 e 5 anos, 21 com mais de 6 anos (30,5\%) e 3 não responderam.

Com relação à participação nas atividades oferecidas: 11 participam de atividades físicas (16\%), enquanto outros 58 não as desenvolvem (84\%); as atividades recreativas atraem a participação de 33 idosos (48\%), porém, delas não participam outros 36 idosos (52\%); 12 participantes desenvolvem atividades voltadas para a educação em saúde (17\%), 57 não despertam interesse nelas (83\%); 37 participam das atividades intelectuais (54\%) e 32 participantes não as desenvolvem (46\%). É importante ressaltar que os idosos podem participar de mais de uma atividade.

As Tabelas l e 2 apresentam os escores médios e o desviopadrão (dp) dos 5 domínios do WHOQOL-BREF e das 6 facetas do WHOQOL-OLD, respectivamente. O domínio com maior escore médio no WHOQOL-BREF é o Psicológico $(15,96)$ seguido da Autoavaliação da Qualidade de Vida (15,94). Já o menor escore médio foi o domínio Ambiental. No WHOQOLOLD o maior escore médio foi o encontrado na faceta que trata do funcionamento do sensório $(15,85)$ e o menor escore médio é a Autonomia $(14,44)$.

Ainda nas Tabelas l e 2 são apresentas, também, a média e desvio-padrão dos grupos de cada variável sociodemográfica pelos domínios ou facetas e, em negrito as variáveis que apresentaram diferenças estatisticamente significativas. Optou-se por apresentar os resultados das variáveis sociodemográficas em que ao menos um domínio ou faceta apresentou diferença estatisticamente significativa.
Tabela 1 - Médias e desvios-padrão (dp) dos grupos das variáveis sociodemográficas nos domínios do WHOQOLBREF. Rio de Janeiro, 2015.

\begin{tabular}{|c|c|c|c|c|c|c|}
\hline & & Fisico & Psicológico & Social & Ambiental & Autoavaliação \\
\hline & & $\begin{array}{l}15,20 \\
(2,51)\end{array}$ & $\begin{array}{l}15,96 \\
(2,10)\end{array}$ & $\begin{array}{l}15,33 \\
(2,87)\end{array}$ & $\begin{array}{l}14,25 \\
(2,37)\end{array}$ & $\begin{array}{l}15,94 \\
(2,46)\end{array}$ \\
\hline \multirow{2}{*}{$\begin{array}{l}\text { Estado } \\
\text { conju- } \\
\text { gal }\end{array}$} & Casados & $\begin{array}{l}15,48 \\
(2,47)\end{array}$ & $\begin{array}{c}16,2 \\
(2,23)\end{array}$ & $\begin{array}{c}15,9 \\
(2,88)\end{array}$ & $\begin{array}{l}14,27 \\
(2,39)\end{array}$ & $\begin{array}{l}16,09 \\
(2,59)\end{array}$ \\
\hline & Não-casados & $\begin{array}{l}14,65 \\
(2,57)\end{array}$ & $\begin{array}{l}15,51 \\
(1,82)\end{array}$ & $\begin{array}{l}14,84 \\
(2,85)\end{array}$ & $\begin{array}{c}14,2 \\
(2,39)\end{array}$ & $\begin{array}{l}15,25 \\
(2,23)\end{array}$ \\
\hline \multirow{3}{*}{$\begin{array}{l}\text { Escola- } \\
\text { ridade }\end{array}$} & $\begin{array}{c}<\text { Funda- } \\
\text { mental }\end{array}$ & $\begin{array}{l}13,77 \\
(2,57)\end{array}$ & $\begin{array}{l}15,45 \\
(2,57)\end{array}$ & $\begin{array}{c}14,0 \\
(3,54)\end{array}$ & $\begin{array}{l}13,85 \\
(2,32)\end{array}$ & $\begin{array}{l}15,25 \\
(2,62)\end{array}$ \\
\hline & Médio & $\begin{array}{l}15,74 \\
(2,17)\end{array}$ & $\begin{array}{l}15,92 \\
(2,31)\end{array}$ & $\begin{array}{l}15,73 \\
(2,88)\end{array}$ & $\begin{array}{l}13,78 \\
(2,21)\end{array}$ & $\begin{array}{c}16,08 \\
(2,2)\end{array}$ \\
\hline & Superior & $\begin{array}{l}15,55 \\
(2,54)\end{array}$ & $\begin{array}{l}16,29 \\
(1,85)\end{array}$ & $\begin{array}{l}15,75 \\
(2,22)\end{array}$ & $\begin{array}{l}14,89 \\
(2,49)\end{array}$ & $\begin{array}{l}16,22 \\
(2,62)\end{array}$ \\
\hline \multirow{3}{*}{$\begin{array}{l}\text { Renda } \\
\text { Própria }\end{array}$} & $1-3 S M$ & $\begin{array}{l}13,84 \\
(2,44)\end{array}$ & $\begin{array}{l}15,43 \\
(2,12)\end{array}$ & $\begin{array}{l}14,47 \\
(2,88)\end{array}$ & $\begin{array}{l}13,62 \\
(1,99)\end{array}$ & $\begin{array}{c}15,3 \\
(2,36)\end{array}$ \\
\hline & $4+S M$ & $\begin{array}{l}14,67 \\
(2,44)\end{array}$ & $\begin{array}{l}15,03 \\
(2,29)\end{array}$ & $\begin{array}{l}15,08 \\
(2,74)\end{array}$ & $\begin{array}{l}14,35 \\
(2,56)\end{array}$ & $\begin{array}{l}15,54 \\
(2,85)\end{array}$ \\
\hline & Não resposta & $\begin{array}{l}16,17 \\
(2,21)\end{array}$ & $\begin{array}{l}16,65 \\
(1,82)\end{array}$ & $\begin{array}{l}15,92 \\
(2,85)\end{array}$ & $\begin{array}{l}14,58 \\
(2,51)\end{array}$ & $\begin{array}{l}16,46 \\
(2,33)\end{array}$ \\
\hline \multirow{4}{*}{ Religião } & Católico & $\begin{array}{l}14,58 \\
(2,56)\end{array}$ & $\begin{array}{c}15,43 \\
(2,2)\end{array}$ & $\begin{array}{l}14,73 \\
(2,83)\end{array}$ & $\begin{array}{l}13,59 \\
(2,18)\end{array}$ & $\begin{array}{l}15,17 \\
(2,28)\end{array}$ \\
\hline & Evangélico & $\begin{array}{l}16,53 \\
(2,07)\end{array}$ & $\begin{array}{l}17,01 \\
(1,49)\end{array}$ & $\begin{array}{l}17,07 \\
(2,21)\end{array}$ & $\begin{array}{l}15,76 \\
(2,37)\end{array}$ & $\begin{array}{c}17,2 \\
(2,11)\end{array}$ \\
\hline & Espirita & $\begin{array}{l}15,89 \\
(2,23)\end{array}$ & $\begin{array}{l}16,91 \\
(1,41)\end{array}$ & $\begin{array}{l}14,93 \\
(2,56)\end{array}$ & $\begin{array}{c}15,1 \\
(0,55)\end{array}$ & $\begin{array}{c}16,8 \\
(1,79)\end{array}$ \\
\hline & Outras & $\begin{array}{c}16,11 \\
(2,66)\end{array}$ & $\begin{array}{l}16,67 \\
(2,49)\end{array}$ & $\begin{array}{l}16,53 \\
(2,92)\end{array}$ & $\begin{array}{c}15,6 \\
(1,92)\end{array}$ & $\begin{array}{c}18 \\
(3,46)\end{array}$ \\
\hline \multirow{2}{*}{$\begin{array}{l}\text { Medica- } \\
\text { mento }\end{array}$} & Sim & $\begin{array}{l}15,99 \\
(2,66)\end{array}$ & $\begin{array}{l}16,89 \\
(1,86)\end{array}$ & $\begin{array}{l}16,67 \\
(2,49)\end{array}$ & $\begin{array}{l}15,35 \\
(1,85)\end{array}$ & $\begin{array}{c}16,0 \\
(2,83)\end{array}$ \\
\hline & Não & $\begin{array}{l}14,99 \\
(2,46)\end{array}$ & $\begin{array}{l}15,71 \\
(2,11)\end{array}$ & $\begin{array}{l}14,99 \\
(2,88)\end{array}$ & $\begin{array}{l}13,96 \\
(2,43)\end{array}$ & $\begin{array}{l}15,93 \\
(2,39)\end{array}$ \\
\hline
\end{tabular}

$\mathrm{Na}$ Tabelal, que correspondem aos valores encontrados para o WHOQOL-BREF, não houve diferença estatisticamente significativa para entre os participantes Casados e Nãocasados, com relação à Escolaridade apenas o domínio Físico apresentou diferença com maior escore médio $(15,74)$ observado nos participantes que possuem ensino médio completo.

Ainda na Tabela 1, as faixas de renda própria apresentam diferenças nos domínios Físico e Psicológico com maiores escores médios entre os participantes que não responderam a que faixa pertenciam (NA), 16,17 e 16,65 respectivamente. Nos domínios Social, Ambiental e Autoavaliação os participantes que se dizem Espíritas apresentam as maiores médias e os menores escores médios são atribuídos aos que se declararam Católicos. Já com relação ao uso de medicamentos existe um escore médio maior do domínio Ambiental aos que afirmam 
fazer uso de medicamento $(15,35)$.

Tabela 2 - Médias e desvios-padrão (dp) dos grupos das variáveis sociodemográficas nos domínios do WHOQOL-OLD. Rio de Janeiro, 2015

\begin{tabular}{|c|c|c|c|c|c|c|c|}
\hline & & $\begin{array}{l}\text { Sen- } \\
\text { sorial }\end{array}$ & $\begin{array}{l}\text { Auto- } \\
\text { nomia }\end{array}$ & $\begin{array}{l}\text { Passado, } \\
\text { presente e } \\
\text { futuro }\end{array}$ & Social & $\begin{array}{c}\text { Morte } \\
\text { e mor- } \\
\text { rer }\end{array}$ & $\begin{array}{l}\text { Intimi- } \\
\text { dade }\end{array}$ \\
\hline & & $\begin{array}{l}15,85 \\
(2,74)\end{array}$ & $\begin{array}{l}14,44 \\
(3,04)\end{array}$ & $14,96(2,54)$ & $\begin{array}{l}14,77 \\
(3,24)\end{array}$ & $\begin{array}{l}14,83 \\
(4,04)\end{array}$ & $\begin{array}{l}14,99 \\
(3.07)\end{array}$ \\
\hline \multirow{2}{*}{$\begin{array}{l}\text { Estado } \\
\text { conju- } \\
\text { gal }\end{array}$} & Casados & $\begin{array}{l}15,98 \\
(2,62)\end{array}$ & $\begin{array}{l}14,86 \\
(3,44)\end{array}$ & $15,11(2,64)$ & $\begin{array}{l}15,07 \\
(3,43)\end{array}$ & $\begin{array}{c}14,6 \\
(4,37)\end{array}$ & $\begin{array}{c}14,8 \\
(3,25)\end{array}$ \\
\hline & $\begin{array}{l}\text { Não-ca- } \\
\text { sados }\end{array}$ & $\begin{array}{l}15,61 \\
(3,01)\end{array}$ & $\begin{array}{r}13,65 \\
(1,97)\end{array}$ & $14,65(2,35)$ & $\begin{array}{l}14,17 \\
(2,82)\end{array}$ & $\begin{array}{c}15,26 \\
(3,4)\end{array}$ & $\begin{array}{l}15,35 \\
(2,76)\end{array}$ \\
\hline \multirow{3}{*}{$\begin{array}{l}\text { Escola- } \\
\text { ridade }\end{array}$} & $\begin{array}{l}<\text { Funda- } \\
\text { mental }\end{array}$ & $\begin{array}{l}14,88 \\
(3,08)\end{array}$ & $\begin{array}{l}14,44 \\
(2,34)\end{array}$ & $15,06(2,36)$ & $\begin{array}{l}13,53 \\
(3,66)\end{array}$ & $\begin{array}{l}14,12 \\
(4,35)\end{array}$ & $\begin{array}{l}14,56 \\
(2,31)\end{array}$ \\
\hline & Médio & $\begin{array}{l}16,42 \\
(2,26)\end{array}$ & $\begin{array}{l}14,42 \\
(2,81)\end{array}$ & $14,68(2,82)$ & $\begin{array}{l}15,12 \\
(3,0)\end{array}$ & $\begin{array}{l}15,84 \\
(3,37)\end{array}$ & $\begin{array}{l}15,36 \\
(3,45)\end{array}$ \\
\hline & Superior & $\begin{array}{l}15,96 \\
(2,85)\end{array}$ & $\begin{array}{l}14,46 \\
(3,69)\end{array}$ & $15,15(2,44)$ & $\begin{array}{l}15,22 \\
(3,11)\end{array}$ & $\begin{array}{l}14,28 \\
(4,39)\end{array}$ & $\begin{array}{l}14,88 \\
(3,18)\end{array}$ \\
\hline \multirow{3}{*}{$\begin{array}{l}\text { Renda } \\
\text { Própria }\end{array}$} & $1-3 S M$ & $\begin{array}{l}15,15 \\
(2,85)\end{array}$ & $\begin{array}{l}13,3 \\
(2,77)\end{array}$ & $14,45(2,58)$ & $\begin{array}{l}13,95 \\
(3,25)\end{array}$ & $\begin{array}{l}14,85 \\
(3,15)\end{array}$ & $\begin{array}{l}15,35 \\
(2,66)\end{array}$ \\
\hline & $4+S M$ & $\begin{array}{l}15,36 \\
(3,25)\end{array}$ & $\begin{array}{l}13,75 \\
(2,26)\end{array}$ & $14,57(2,38)$ & $\begin{array}{c}14,36 \\
(2,5)\end{array}$ & $\begin{array}{l}13,62 \\
(4,54)\end{array}$ & $\begin{array}{l}15,15 \\
(3,0)\end{array}$ \\
\hline & $\begin{array}{l}\text { Não } \\
\text { resposta }\end{array}$ & $\begin{array}{l}16,47 \\
(2,38)\end{array}$ & $\begin{array}{l}15,35 \\
(3,22)\end{array}$ & $15,4(2,57)$ & $\begin{array}{c}15,4 \\
(3,45)\end{array}$ & $\begin{array}{c}15,3 \\
(4,33)\end{array}$ & $\begin{array}{l}14,71 \\
(3,38)\end{array}$ \\
\hline \multirow{4}{*}{ Religião } & Católico & $\begin{array}{l}15,45 \\
(2,63)\end{array}$ & $\begin{array}{c}14 \\
(3,33)\end{array}$ & $14,81(2,57)$ & $\begin{array}{l}14,48 \\
(3,28)\end{array}$ & $\begin{array}{l}13,88 \\
(4,11)\end{array}$ & $\begin{array}{c}14,63 \\
(2,8)\end{array}$ \\
\hline & $\begin{array}{l}\text { Evangé- } \\
\text { lico }\end{array}$ & $\begin{array}{l}15,8 \\
(2,76)\end{array}$ & $\begin{array}{l}15,2 \\
(2,31)\end{array}$ & $15,6(1,64)$ & $\begin{array}{l}15,53 \\
(2,92)\end{array}$ & $\begin{array}{l}16,79 \\
(3,31)\end{array}$ & $\begin{array}{l}16,29 \\
(1,64)\end{array}$ \\
\hline & Espirita & $\begin{array}{l}17,75 \\
(3,2)\end{array}$ & $\begin{array}{l}15,25 \\
(2,22)\end{array}$ & $14,8(3,29)$ & $\begin{array}{c}15,2 \\
(3,19)\end{array}$ & $\begin{array}{c}17,8 \\
(2,68)\end{array}$ & $\begin{array}{c}13,4 \\
(5,37)\end{array}$ \\
\hline & Outras & $\begin{array}{l}17,75 \\
(2,95)\end{array}$ & $\begin{array}{l}15,5 \\
(3,87)\end{array}$ & $15,8 \quad(3,7)$ & $\begin{array}{l}16,0 \\
(3,81)\end{array}$ & $\begin{array}{c}14,8 \\
(4,55)\end{array}$ & $\begin{array}{c}17,4 \\
(3,58)\end{array}$ \\
\hline \multirow{2}{*}{$\begin{array}{l}\text { Medica- } \\
\text { mento }\end{array}$} & Sim & $\begin{array}{c}17,0 \\
(2,72)\end{array}$ & $\begin{array}{l}14,29 \\
(3,07)\end{array}$ & $15,79(2,81)$ & $\begin{array}{l}16.07 \\
(3,0)\end{array}$ & $\begin{array}{c}15,79 \\
(3,4)\end{array}$ & $\begin{array}{c}16,0 \\
(2,88)\end{array}$ \\
\hline & Não & $\begin{array}{c}15,56 \\
(2,7)\end{array}$ & $\begin{array}{l}14,48 \\
(3,07)\end{array}$ & $14,75 \quad(2,45)$ & $\begin{array}{l}14,44 \\
(3,25)\end{array}$ & $\begin{array}{l}14,58 \\
(4,19)\end{array}$ & $\begin{array}{c}14,72 \\
(3,1)\end{array}$ \\
\hline
\end{tabular}

Na Tabela 2 percebe-se que os casados apresentam maior escore médio $(14,86)$ na faceta Autonomia. Não há diferença estatisticamente significativa entre os diferentes níveis de escolaridade. Os que não responderam a faixa de renda própria apresentam o maior escore médio (15,35). Já com relação à religião os Espíritas apresentam maior escore médio para a faceta Morte e Morrer, entretanto, para a faceta Intimidade este mesmo grupo apresenta o menor escore médio $(13,4)$. O uso de medicamento não apresentou diferença significativa para nenhuma das facetas.

Com relação às variáveis sociodemográfica, a saber: Sexo, Idade e DCNT, bem como o tempo de participação nas atividades do centro de lazer não apresentaram diferenças estatisticamente significativa. Já para as atividades desenvolvidas no centro apenas as atividades Intelectuais apresentaram diferença estatisticamente significativa no domínio Ambiental, com escore médio de 14,84 (sd = 2,49) para os que afirmam participarem destas atividades contra 13,74 (sd = 2,18) para os que não fazem atividades Intelectuais. DISCUSSÃO

O envelhecimento populacional é um fenômeno universal e poderá tornar o Brasil o sexto colocado no ranking dos países com mais idosos no mundo, quantificando, em 2025 , cerca de 32 milhões de idosos ${ }^{(12)}$

Nada obstante a reiteração censitária do envelhecimento da população, é importante destacar que a participação dos idosos em grupos de convivência tem aumentado no Brasil, considerando que esta favorece a promoção de saúde na terceira idade ${ }^{(13)}$.

Considerando o envelhecimento mundial da população e a importância de se concentrar esforços para atender as necessidades de saúde desse segmento, estudo realizado na Korea ${ }^{(14)}$ visou avaliar como a qualidade de vida está relacionada com sintomas comuns experimentados pelos idosos, incluindo fadiga, dor, distúrbios do sono, indigestão e depressão.

Na mesma sintonia de preocupação com o envelhecimento populacional, pesquisadores investem em estudos sobre promoção da qualidade de vida dos Idosos em Kuala Lumpur, Malásia(15), na Austrália(16), na Suiça ${ }^{(17)}$, no Canadá ${ }^{(18)}$ e no México(19), como forma de elaborar diagnóstico situacional que viabilize o planejamento, implementação e avaliação de estratégias de ação condizentes com a crescente demanda por serviços especializados na área geriátrica.

Sabe-se que o envelhecimento populacional brasileiro está relacionado ao acúmulo de incapacidades progressivas nas atividades funcionais e de vida diárias, cuja mortalidade é substituída por comorbidades. A manutenção da capacidade funcional dos idosos ${ }^{(7)}$ surge como como novo e relevante paradigma de saúde ${ }^{(20)}$.

A propósito da relação funcionalidade e fragilidade na idade avançada, resultados do estudo realizado com 117 idosos cadastrados na Regional Norte da Prefeitura de Belo Horizonte, Estado de Minas Gerais, no período de agosto de 2009 a junho de 2010 , demonstraram maior número de idosos pré-frágeis e uma associação entre o perfil de fragilidade e a funcionalidade ${ }^{(12)}$

A adoção de medidas preventivas às complicações no quadro de saúde dos idosos é fundamental(21). Assim, as atividades de educação em saúde oferecidas nos centros de convivência devem ser da competência dos enfermeiros e demais profissionais da área de saúde, e focadas em oportunidades para que as pessoas idosas possam escolher por estilos de vida saudáveis, dentro de suas próprias expectativas e condições de saúde ${ }^{(22)}$. Essa premissa pode ter faltado aos profissionais de saúde que atuam junto aos idosos deste estudo, considerando a baixa adesão dos participantes no programado pelas atividades de educação em saúde, apenas (17,39\%). Além da ausência de significância estatística nos domínios e facetas entre os que participam das atividades do grupo de convivência. 
Quanto à questão de gênero, estudo realizado com 85 idosos participantes de grupos de convivência, em cidade da região norte do Estado do Rio Grande do Sul, revelou predomínio de mulheres, como nesta investigação. O que confirma maior prevalência de mulheres em comparação ao número de homens idosos que frequentam programas similares ${ }^{(10)}$

Estudo realizado em seis grupos de convivência da cidade de Cajazeiras-PB(20), Brasil, no periodo de setembro a outubro de 2010 , identificou a predominância de mulheres em relação aos homens, o que pode estar relacionado à maior expectativa de vida por parte da população feminina. Identificou-se também a resistência masculina, que por sua vez foi um fator significativo, pois quando perguntadas pelos esposos, as casadas respondiam que os companheiros, por preconceito, não as acompanhavam aos encontros.

De acordo com estudo realizado no Estado do Rio Grande do Sul(10), a motivação feminina em participar das atividades de lazer é diferente da masculina, uma vez que a maior parte das idosas atuais exerceu um papel restrito ao ambiente doméstico.

O envelhecimento não deve ser percebido como fase da vida em que as pessoas não mais estão aptas ao aprendizado, ao contrário, cabe enfatizar que anos adicionais de vida oferecem a oportunidade aos idosos de desenvolverem novas atividades, tais como a educação, uma nova carreira ou buscar desenvolver algo que tenha sido negligenciado ao longo da vida $^{(23)}$. Algo percebido entre importante contingente de participantes deste estudo, considerando que 53,62\% prefere se dedicar às atividades intelectuais no centro de lazer, pelo que elas representam na ampliação do conhecimento sobre a vida, a saúde e o próprio processo de envelhecimento.

É essencial que os gestores públicos assumam que idosos participando efetivamente de atividades físicas orientadas por profissionais especializados, reduz gastos maiores em decorrência de complicações de doenças crônicas não transmissiveis, como informadas por $60,67 \%$ dos participantes deste estudo. Assim, faz mister difundir que a prática de exercícios físicos entre os idosos é uma estratégia simples, barata e eficaz, tanto para diminuir os custos relacionados à saúde, quanto para melhorar a qualidade de vida(24).

Qualidade de vida na idade avançada requer particularmente de equilibrio entre o declínio natural das diversas capacidades individuais, mentais e físicas e o alcance do que se almeja através de estratégias propostas pelos profissionais da saúde em parceria com a pessoa idosa, a familia e a comunidade ${ }^{(22)}$.

Similar aos resultados deste estudo, no referente as faixas etárias dos participantes em centros de lazer e convivência, a baixa participação de idosos entre 75 e 84 anos, percebida em investigação realizada no interior do Estado da Paraíba pode estar relacionada ao grau de dependências e comorbidades, devido ao aumento da idade, pois esses fatores podem limitar o acesso e a participação dos idosos em encontros com o grupo $^{(20)}$.

Um achado importante do estudo realizado com 64 idosos da comunidade que desenvolvem atividades em grupos de convivência de Itabira-MG, está relacionado ao número de medicamentos usados. Constatou-se o uso regular de medicamentos por $60 \%$ dos idosos ${ }^{(13)}$, corroborando com os achados deste estudo, pois $79,71 \%$ participantes relataram fazer uso sistemático de algum medicamento para controle de doença crônica não transmissivel.

A faceta Morte e Morrer apresentada por idosos Espíritas pode ser explicada por meio da própria religiosidade que, como sistemas de crenças, influi na percepção e compreensão dos idosos sobre as experiências com perdas, sofrimento e dor vivenciadas no envelhecimento. Como suporte para as questões existenciais, oferece mais sentido à vida e à morte pela compreensão da finitude do ser humano, contribuindo para promoção da sua saúde mental e física ${ }^{(25)}$.

Apesar das dificuldades e da própria resistência para aprender as novas tecnologias de comunicação, os idosos têm frequentado aulas de informática com a finalidade de se tornarem usuários de computador, mostrando-se mais predispostos a se divertir, além de se relacionarem com os colegas e parentes através dos meios de socialização digitais mais comuns(23). No caso deste estudo, o atípico nivel de escolaridade dos seus participantes constitui fator decisivo para que predomine habilidade de fazer uso desses recursos para melhor interação com o universo de informações disponíveis em seus computadores domésticos e equipamentos móveis de comunicação.

Acompanhando o fenômeno de envelhecimento da população, a Organização Mundial da Saúde (OMS) desenvolve projetos que objetivam a sensibilização e a educação dos profissionais da Atenção Primária à Saúde (APS) para que estes possam atuar diante das necessidades dos idosos. Diante do atual quadro de envelhecimento populacional, o importante é que as equipes de saúde da APS estejam treinadas e preparadas para o implemento de ações preventivas de agravos e promoção da OV dessas pessoas ${ }^{(26)}$.

\section{Limitações do estudo}

Por se tratar de um estudo transversal, realizado em único Centro de Lazer para Idosos, os resultados devem ser tratados com certa cautela. Apesar de o estudo ter revelado característica sociodemográficas e a mensuração da qualidade de vida dos idosos, carece de outras investigações em contextos afins para se considerar com maior grau de certeza se a relação entre eles é causal ou não. 


\section{Contribuições do estudo para a prática}

Este estudo contribui para nortear as intervenções dos profissionais de saúde atuantes em programas institucionais de lazer e inclusão de idosos, sendo também de grande valia para a prática da assistência de Enfermagem focada na promoção da saúde e qualidade de vida dessas pessoas. Afinal, há que se considerar que vivemos no contexto de uma sociedade com crescentes taxas de longevidade.

\section{CONCLUSÃO}

O grupo de convivência de idosos é uma importante ferramenta de promoção da saúde e qualidade de vida desta população e no UnATI/UERJ são oferecidas atividades físicas, recreativas, intelectuais, de educação em saúde, entre outros.

A religião e a faixa de renda própria são variáveis sociodemográfica que alteram a percepção da qualidade de vida, isto pode ser observado nas médias dos domínios e facetas.
Os resultados deste estudo descrevem característica sociodemográficas e a mensuração da qualidade de vida dos idosos que participam de grupo de convivência e estas informações fornecem subsidios para aprimorar a atuação dos profissionais de saúde interessados em executar práticas proativas de promoção de qualidade de vida e saúde dos idosos.

\section{Contribuição dos autores}

RAS e WCAM, concepção, desenho, análise e interpretação dos dados, redação do artigo, revisão crítica, revisão final. NMAF, ASS e TT, análise e interpretação dos dados, redação do artigo, revisão crítica. SSB, AAM e ACSS redação do artigo, revisão crítica, revisão final. 


\section{REFERÊNCIAS}

1. Instituto Brasileiro de Geografia e Estatística (IBGE). Estudos e Pesquisas. Informação Demográfica e Socioeconômica, número 29. Sintese de Indicadores Sociais. Uma análise das condições de vida da população brasileira. Rio de Janeiro: IBGE [Internet]. 2012 [cited 2017 Nov 12]. Available from: http://biblioteca.ibge.gov. br/visualizacao/livros/liv62715.pdf

2. Serbim AK, Figueiredo AEPL. Qualidade de vida de idosos em um grupo de convivência. Sci. med. (Porto Alegre, Online) [Internet]. 2011 [cited 2018 Feb 14]; 21(4): 166-72 Available from: http://revistaseletronicas.pucrs.br/ojs/index.php/scientiamedica/article/download/9405/7237

3. Braga MCP. Casella MA, Campos MLN. Paiva SP. Qualidade de vida medida pelo WHOQOL-BREF: Estudo com idosos residentes em Juiz de fora/MG. Rev. APS. [Internet]. 2011 [cited 2017 Nov 18]; 14(1): 93-100. Available from: https://aps.ufjf. emnuvens.com.br/aps/article/view/965/450

4. Yassuda MS, Silva HS da. Participação em programas para a terceira idade: impacto sobre a cognição, humor e satisfação com a vida. Estud. Psicol. (Campinas, Online). [Internet]. 2010 [cited 2018 Feb 11]; 27(2): 207-14. Available from: http://www.scielo.br/scielo.php?script=sci_arttext\&pi$d=S 0103-166 \times 2010000200008 \varepsilon \operatorname{lng}=p t \delta n r m=i s o \&$ tlng $=p t$

5. OMS. Organização mundial da Saúde. Envelhecimento ativo: uma politica de saúde (Suzana Montijo, trad.). Brasilia: Organização Pan-Americana da Saúde, 2005.

6. Campos ACV, Cordeiro EC, Rezende GP de, Vargas AD, Ferreira EF e. Quality of life of elderly practitioners of physical activity in the context of the family health strategy. Texto \& contexto enferm. (Online) [Internet]. 2014 [cited 2018 May 05] 23(4): 889-97. Available from: http://www.scielo.br/scielo.php?script=sci_arttextEpid =S0104-07072014000400889\&lng=pt\&nrm=iso\&tlng=en

7. Goes TM. Polaro SHI, Gonçalves LHT. Cultivo do bem viver das pessoas idosas e tecnologia cuidativo-educacional de enfermagem. Enferm Foco [Internet]. 2016 [cited 2018 Jan 28]; 7(2): 47-51. Available from: http://revista.cofen.gov.br/index. php/enfermagem/article/view/794/319

8. Machado WCA, Figueiredo NMA, Miranda RS, Shubert CO. Domestic violence against the elderly: reflections on assistance and nursing care. J Nurs UFPE (Online) [Internet]. 2013 (cited 2018 Jan 18]; 7(12):6936-41. Available from: https://periodicos.ufpe.br/revistas/revistaenfermagem/article/view/12360/15094

9. Vitorino LM, Paskulin LMG, Vianna LAC. Quality of life of seniors living in the community and in long term care facilities: a comparative study. Rev. latinoam. enferm [Internet]. 2013 [cited 2017 Dec 03]; 21(n.esp):
3-11. Available from: http://www.scielo.br/scielo.php?script=sci_arttext8pi3-11. Available from: http:// www. scielo.br/scielo.ph
$\mathrm{d}=\mathrm{S} 0104-116920130007000028$ lng $=$ pt\&nrm=iso\&tlng=en

10. Leite MT, Hilderbrandt LM, Kirchner RM, Winck MT, Silva AA. Franco GP. Estado cognitivo e condições de saúde de idosos que participam de grupos de convivência. Rev. gaúcha enferm. (Online) [Internet]. 2012 [cited 2017 Nov 14]: 33(4): 64-71. Available from: http://www.scielo.br/scielo.php?script=sci_arttext\&pi$d=S 1983-14472012000400008 \& \operatorname{lng}=p t \& n r m=i s o$

11. Campos ACV, Cordeiro EC, Rezende GP de, Vargas AD, Ferreira EF e. Quality of life of elderly practitioners of physical activity in the context of the family health strategy. Texto \& contexto enferm. (Online) [Internet]. 2014 [cited 2017 Nov 11]: 23(4): 889-97. Available from: http://www.scielo.br/scielo.php?script=sci_arttextEpid=S0104-07072014000400889\&lng=pt\&nrm=iso\&tlng=en

12. Lustosa LP, Marra TA, Pessanha FPAS, Freitas JC, Guedes RC. Fragilidade e funcionalidade entre idosos frequentadores de grupos de convivência em Belo Horizonte, MG. Rev. bras. geriatr. gerontol. (Online). [Internet]. 2013 [cited 2018 Feb 09]: 16(2): 347-54. Available from: http://www.scielo.br/scielo.php?script=sci_arttext8pid=S1809-982320130002000148lng=ptEnrm=iso

13. Carmo LV, Drummond LP. Arantes PMA. Avaliação do nivel de fragilidade em idosos participantes de um grupo de convivência. Fisioter. Pesqui. (Online). [Internet]. 2011 [cited 2017 Nov 21]; 18(1): 17-22. Available from: http://www.scielo.br/scielo. php?script=sci_arttext\&pid=S1809=29502011000100004-\&lng=pt\&nrm=iso\&tlngpt

14. Mun S. Park K. Baek Y, Lee S, Yoo J. Interrelationships among common symptoms in the elderly and their effects on health-related quality of life: a cross-sectional study in rural Korea. Health and Quality of Life Outcomes [Internet]. 2016 tional study in rural Korea. Health and Quality of Life Outcomes [Internet]. 2016
[cited 2017 Dec 18]; 14(146). Available from: http://hqlo.biomedcentral.com/arti[cited 2017 Dec 18]; 14(146). Availal
cles/10.1186/s12955-016-0549-9
15. Onunkwor OF, Al-Dubai SAR, George PP, Arokiasamy J, Yadav H, Barua A, Shuaibu HO. A cross-sectional study on quality of life among the elderly in non-governmental organizations' elderly homes in Kuala Lumpur. Health and Quality of Life Outcomes [Internet]. 2016 [cited 2018 Jan 13]; 14(6). Available from: http://hqlo. biomedcentral.com/articles/10.1186/s12955-016-0408-8

16. Welsh J, Strazdins L, Charlesworth S, Kulik CT, Butterworth P. Health or harm? A cohort study of the importance of job quality in extended workforce participation by older adults. Public Health. BMC [Internet]. 2016 [cited 2017 Dec 18]; 16(1): 885. Available from: https://www.ncbi.nlm.nih.gov/pmc/articles/PMC5000457/?tool=pubmed

17. Bernard M. Braunschweig G. Fegg MJ, Borasio GD. Meaning in life and perceived quality of life in Switzerland: results of a representative survey in the German. French and Italian regions. Health and Quality of Life Outcomes [Internet]; 2015 [cited 2017 Dec 19]; 13(160). Available from: http://hqlo.biomedcentral.com/articles/10.1186/s12955-015-0353-y

18. Davis JC. Bryan S, Best JR, Li LC. Hsu CL, Gomez et al. Mobility predicts change in older adults' health-related quality of life: evidence from a Vancouver falls prevention prospective cohort study. Health and Quality of Life Outcomes [Internet]. 2015 [cited 2017 Dec 19]; 13(101). Available from: http://hqlo.biomedcentral.com 2015 [cited 2017 Dec 19]; 13(101). Availa
articles/10.1186/s12955-015-0299-0

19. Loredo-Figuerosa MT, Gallegos-Torres RM. Xeque-Morales AS, Palomé-Veja G. Juárez-Lira A. Nivel de dependência, autocuidado y calidad de vida del adulto mayor. Enferm. univ. [Internet]. 2016 [cited 2018 Jan 16]: 13(3): 159-65. Available from: http://www.revistas.unam.mx/index.php/reu/article/view/56819/50422

20. Andrade NA, Nascimento MMP, Oliveira MMD, Queiroga RM, Fonseca FLA, Adami F. Percepção de idosos sobre grupo de convivência: estudo na cidade de Cajazeiras-PB. Rev. bras. geriatr. gerontol. (Online) [Internet]. 2014 [cited 2018 Feb 14] 17(1): 39-48. Available from: http://www.scielo.br/scielo.php?script=sci_arttext\&pid=S1809-98232014000100039\&lng=pt\&nrm=iso

21. Pagliosa LC, Renosto A. Effects of a health promotion and fall prevention program in elderly individuals participating in interaction groups. Fisioter. Mov. (Online). [Internet]. 2014 [cited 2018 Jan 20]: 27(1): 101-09. Available from: . . http://www.scielo $\mathrm{br} /$ scielo.php?script $=$ sci_arttext\&pid $=S 0103=51502014000100101-\delta \operatorname{lng}=p t \delta \mathrm{nrm}^{-}$ iso

22. Ilha S, Argenta C, Silva MRS da, Cezar-Vaz MR, Pelzer MT, Backes DS. Active aging: necessary reflections for nurse/health professionals. Rev. pesqui. cuid. ve aging: necessary reflections for nurse/health professionals. Rev. pesqui. cuid. fundam. (Ontine). [Internet] 2016 [cited 2018 Aug 18]; 8(2): 4231-42, Available fror
http://www.seer.unirio.br/index.php/cuidadofundamental/article/view/4242

23. Carvalho E, Arantes RC, Cintra ASR. The inclusion of elderly persons from the Instituto Henrique da Silva Semente (IHESS) in Indaiatuba, São Paulo, in the digital age: physio-gerontological contributions. Rev. bras. geriatr. gerontol. (Online) [Internet]. 2016 [cited 2018 May 28]: 19(4): 567-75. Available from: http://www. scielo. $\mathrm{br} /$ scielo.php?script=sci_arttext\&pid=S1809-98232016000400567

24. Salin MS, Mazo GZ, Cardoso AS, Garcia GS. Atividade fisica para idosos: diretrizes para implantação de programas e ações. Rev. bras. geriatr. gerontol. (Online) [Internet]. 2011 [cited 2018 Jul 12]: 14(2): 197-208. Available from: http://www.scielo.br/scielo.php?script=sci_arttext\&pi$\mathrm{d}=$ S1809-982320110002000028lng=pt\&nrm=iso\&tlng=pt

25. Machado WCA, Leite JL. Eros e Thanatos: A morte sob a ótica da enfermagem. São Caetano do Sul: Difusão. 2004. 224 p.

26. Witt RR, Roos MO, Carvalho NM. Silva AM. Rodrigues CDS, Santos MT dos. Professional competencies in primary health care for attending to older adults. Rev. Esc. Enferm. USP [Internet]. 2014 [cited 2018 Aug 15]: 48(6): 120-25. Available from: http://www.scielo.br/scielo.php?script=sci_arttext\&pi$\mathrm{d}=50080-623420140006010208 \operatorname{lng}=$ pt\&nrm=iso\&tlng=en 